\title{
The solvability of nonhomogeneous boundary value problems with $\phi$-Laplacian operator
}

\author{
Sha-Sha Chen ${ }^{1}$ and Zhi-Hong Ma 2* $^{*}$
}

\section{"Correspondence:}

mazhihong_8@163.com

${ }^{2}$ Basic Science Department, Tianjin

Agricultural University, Tianjin,

300384, P.R. China

Full list of author information is

available at the end of the article

\begin{abstract}
We treat the nonhomogeneous boundary value problems with $\phi$-Laplacian operator $\left(\phi\left(u^{\prime}(t)\right)\right)^{\prime}=-f\left(t, u(t), u^{\prime}(t)\right), t \in(0, T), u(0)=A, \phi\left(u^{\prime}(T)\right)=\tau u(T)+\sum_{j=1}^{k} \tau_{i} u\left(\zeta_{i}\right)$, where $\phi:(-a, a) \rightarrow(-b, b)(0<a, b \leq+\infty)$ is an increasing homeomorphism such that $\phi(0)=0, \tau, \tau_{i} \in \mathbb{R}, \zeta_{i} \in(0, T), i=1,2, \ldots, k, A \geq 0$, and $f:[0, T] \times \mathbb{R} \times \mathbb{R} \rightarrow \mathbb{R}$ is continuous. We will show that even if some of the $\tau$ and $\tau_{i}$ are negative, the boundary value problem with singular $\phi$-Laplacian operator is always solvable, and the problem with a bounded $\phi$-Laplacian operator has at least one positive solution.
\end{abstract}

Keywords: nonhomogeneous; $\phi$-Laplacian; negative coefficient; positive solution

\section{Introduction}

We are concerned with the nonhomogeneous boundary value problem with $\phi$-Laplacian operator

$$
\left\{\begin{array}{l}
\left(\phi\left(u^{\prime}(t)\right)\right)^{\prime}=-f\left(t, u(t), u^{\prime}(t)\right), \quad t \in(0, T), \\
u(0)=A, \quad \phi\left(u^{\prime}(T)\right)=\tau u(T)+\sum_{i=1}^{k} \tau_{i} u\left(\zeta_{i}\right),
\end{array}\right.
$$

where $\phi:(-a, a) \rightarrow(-b, b)(0<a, b \leq+\infty)$ is an increasing homeomorphism such that $\phi(0)=0, \tau, \tau_{i} \in \mathbb{R}, \zeta_{i} \in(0, T), i=1,2, \ldots, k, A \geq 0$, and $f:[0, T] \times \mathbb{R} \times \mathbb{R} \rightarrow \mathbb{R}$ is continuous.

According to the related literature [1-5], a $\phi$-Laplacian operator is said to be singular when the domain of $\phi$ is finite (i.e., $a<+\infty$ ), on the contrary the operator is called regular. On the other hand, we say that $\phi$ is bounded if its range is finite $(i . e ., b<+\infty)$ and unbounded in other case. There are three paradigmatic models in this context:

(1) $a=b=+\infty$ (regular unbounded): The $p$-Laplacian operator

$$
\phi_{p}(x)=|x|^{p-2} x, \quad \text { with } p>1 .
$$

(2) $a<+\infty, b=+\infty$ (singular unbounded): The relativistic operator

$$
\phi(x)=\frac{x}{\sqrt{1-x^{2}}} .
$$

(3) $a=+\infty, b<+\infty$ (regular bounded): The one dimensional mean curvature operator

$$
\phi(x)=\frac{x}{\sqrt{1+x^{2}}} .
$$

O2014 Chen and Ma; licensee Springer. This is an Open Access article distributed under the terms of the Creative Commons Attribution License (http://creativecommons.org/licenses/by/2.0), which permits unrestricted use, distribution, and reproduction in any medium, provided the original work is properly cited. 
The study of the $\phi$-Laplacian equations is a classical topic that has attracted the attention of many experts because of its interest in applications. Since 2004, in a number of papers, Bereanu and Mawhin have considered such problems with Dirichlet, Neumann or periodic boundary conditions (see, for example, [1-3] and the references therein). In these papers, the various boundary value problems are reduced to the search for fixed point of some nonlinear operators defined on Banach spaces. In particular, they have also considered some boundary value problems with nonhomogeneous boundary conditions, and they obtained the existence of solutions by the use of the Schauder fixed point theorem (see [2, 3]). Recently, Torres [4] proved the existence of a solution of a forced Liénard differential equation with $\phi$-Laplacian by means of Schauder fixed point theorem.

We note here that many nonlinear differential problems require the search of positive, meaningful, solutions. The existence of positive solutions for ordinary differential equations and $p$-Laplacian equations have been studied by several authors and many interesting results has been obtained (only to mention some of them; see [5-7], and the references therein). If the coefficient occurring in the boundary conditions takes a negative value, then the existence of a positive solution for a BVP with $\phi$-Laplacian operator is less considered because it is sometimes difficult to construct a corresponding cone for applying the fixed point theorem.

The purpose of this paper is to study the nonhomogeneous boundary value problem (1.1) with $\phi$-Laplacian operator even in the case where some of the $\tau$ and $\tau_{i}$ are negative. Firstly, we show that the problem with singular $\phi$-Laplacian operator is always solvable by the use of Schauder fixed point theorem. Secondly, we prove that the problem with bounded $\phi$-Laplacian operator has at least one positive solution by means of a change of variable and the Krasnosel'skii fixed point theorem. As we will see, the interesting points of the paper are the following:

(1) Some of the $\tau$ and $\tau_{i}$ coefficients in (1.1) are allowed to take a negative value.

(2) In order to obtain a positive solution of the problem (1.1), we make a change of variable and generate two first-order differential equations and the corresponding nonlinear operator $B$. The new method can be used for the differential domains and ranges of $\phi^{-1}$ and give an a priori estimate of the solution.

This paper is organized as follows. In Section 2, we give some preliminaries and lemmas. In Section 3, we show some theorems on the existence of (positive) solutions of the differential equations (1.1). Moreover, an example is given to illustrate our results.

\section{Preliminaries and lemmas}

Firstly, we consider the following $\phi$-Laplacian differential equation:

$$
\left\{\begin{array}{l}
\left(\phi\left(u^{\prime}(t)\right)\right)^{\prime}=-h(t), \quad t \in(0, T), \\
u(0)=A, \quad \phi\left(u^{\prime}(T)\right)=\tau u(T)+\sum_{i=1}^{k} \tau_{i} u\left(\zeta_{i}\right),
\end{array}\right.
$$

where $h \in L_{1}[0, T]$.

Next we make the change of variable

$$
x(t)=\phi\left(u^{\prime}(t)\right),
$$

i.e.,

$$
u^{\prime}(t)=\phi^{-1}(x(t)) .
$$


Together with (2.1), we get the following two first-order differential equations:

$$
\left\{\begin{array}{l}
u^{\prime}(t)=\phi^{-1}(x(t)) \\
u(0)=A
\end{array}\right.
$$

and

$$
\left\{\begin{array}{l}
x^{\prime}(t)=-h(t), \\
x(T)=\tau u(T)+\sum_{i=1}^{k} \tau_{i} u\left(\zeta_{i}\right) .
\end{array}\right.
$$

Integration in (2.4) yields

$$
u(t)=A+\int_{0}^{t} \phi^{-1}(x(s)) d s
$$

Then we get

$$
u(T)=A+\int_{0}^{T} \phi^{-1}(x(s)) d s, \quad u\left(\zeta_{i}\right)=A+\int_{0}^{\zeta_{i}} \phi^{-1}(x(s)) d s .
$$

Thus, together with the boundary condition in (2.5), we obtain

$$
\begin{aligned}
x(T) & =\tau u(T)+\sum_{i=1}^{k} \tau_{i} u\left(\zeta_{i}\right) \\
& =A \sum_{i=1}^{k+1} \tau_{i}+\sum_{i=1}^{k+1} \tau_{i} \int_{0}^{\zeta_{i}} \phi^{-1}(x(s)) d s,
\end{aligned}
$$

where we denote $\tau_{k+1}:=\tau$ and $\zeta_{k+1}:=T$. Thus (2.5) can be rewritten as follows:

$$
\left\{\begin{array}{l}
x^{\prime}(t)=-h(t), \\
x(T)=A \sum_{i=1}^{k+1} \tau_{i}+\sum_{i=1}^{k+1} \tau_{i} \int_{0}^{\zeta_{i}} \phi^{-1}(x(s)) d s .
\end{array}\right.
$$

Integration leads to

$$
\begin{aligned}
x(t) & =x(T)+\int_{t}^{T} h(s) d s \\
& =A \sum_{i=1}^{k+1} \tau_{i}+\sum_{i=1}^{k+1} \tau_{i} \int_{0}^{\zeta_{i}} \phi^{-1}(x(s)) d s+\int_{t}^{T} h(s) d s .
\end{aligned}
$$

Now, we define two nonlinear operators $B$ and $S$, respectively, by

$$
\begin{aligned}
(B x)(t):= & A \sum_{i=1}^{k+1} \tau_{i}+\sum_{i=1}^{k+1} \tau_{i} \int_{0}^{\zeta_{i}} \phi^{-1}(x(s)) d s \\
& +\int_{t}^{T} f\left(s, A+\int_{0}^{s} \phi^{-1}(x(\tau)) d \tau, \phi^{-1}(x(s))\right) d s
\end{aligned}
$$


and

$$
(S u)(t):=A+\int_{0}^{t} \phi^{-1}((B x)(s)) d s .
$$

Remark 2.1 From the deduction of (2.2)-(2.7), we find that if $\phi^{-1}(x)$ is well defined and $x$ is a fixed point of the nonlinear operator $B$, then $u(t)$ in (2.6) is a solution of the problem (1.1).

To such a continuous function $f$, we associate its Nemytskii operator $N_{f}: C^{1} \rightarrow C$ defined by

$$
N_{f}(u)(t)=f\left(t, u(t), u^{\prime}(t)\right), \quad t \in[0, T] .
$$

It is easy to verify that $N_{f}$ is continuous and takes bounded sets into bounded sets.

Lemma 2.1 (See [8]) Let $X$ be a Banach space and $P \subseteq X$ a cone. Suppose that $\Omega_{1}$ and $\Omega_{2}$ are bounded open sets contained in $X$ such that $0 \in \Omega_{1}$ and $\bar{\Omega}_{1} \subseteq \Omega_{2}$. Suppose further that $S: P \cap\left(\bar{\Omega}_{2} \backslash \Omega_{1}\right) \rightarrow P$ is a completely continuous operator. If either

(i) $\|S u\| \leq\|u\|$ for $u \in P \cap \partial \Omega_{1}$ and $\|S u\| \geq\|u\|$ for $u \in P \cap \partial \Omega_{2}$, or

(ii) $\|S u\| \geq\|u\|$ for $u \in P \cap \partial \Omega_{1}$ and $\|S u\| \leq\|u\|$ for $u \in P \cap \partial \Omega_{2}$, then $S$ has at least one fixed point in $P \cap\left(\bar{\Omega}_{2} \backslash \Omega_{1}\right)$.

\section{The main result}

Let $X$ be the Banach space $C([0, T])$ with the maximum norm $\|x\|=\max _{t \in[0, T]}|x(t)|$. Define a cone by

$$
P=\{x \in C([0, T]): x(t) \geq 0\} .
$$

For the sake of convenience, we give the following conditions:

(i) The nonlinearity $f:[0, T] \times[A-a T, A+a T] \times(-a, a) \rightarrow \mathbb{R}^{+}$satisfies

$$
0 \leq f(t, u, v) \leq M
$$

for any $(t, u, v) \in[0, T] \times[A-a T, A+a T] \times(-a, a)$;

(ii) $\Delta=A \sum_{i=1}^{k+1} \tau_{i}>0$;

(iii) There exists a positive constant $r$ such that

$$
\Delta+\lambda \phi^{-1}(r)+M T \leq r<b,
$$

where $\lambda=\sum_{i \in \Gamma} \tau_{i} \zeta_{i}, \Gamma=\left\{i: \tau_{i} \geq 0\right\}$

(iv) $\min _{0 \leq v(t)<b} \sum_{i=1}^{k+1} \tau_{i} \int_{0}^{\zeta_{i}} \phi^{-1}(v(s)) d s \geq 0$.

\section{Remark 3.1}

(1) If $\tau_{i} \geq 0$, for all $i=1,2, \ldots, k+1$, then the condition (iv) clearly holds.

(2) If $A=0$, instead of conditions (i) and (ii), we assume that for any

$(t, u, v) \in[0, T] \times[A-a T, A+a T] \times(-a, a)$, the following inequalities hold:

$$
M_{1} \leq f(t, u, v) \leq M_{2},
$$


where $0<M_{1}<M_{2}$ are two constants. Also, the condition (3.1) is replaced by

$$
\lambda \phi^{-1}(r)+M_{2} T \leq r<b .
$$

Case I. Singular $\phi$-Laplacian operator: $\phi:(-a, a) \rightarrow(-\infty,+\infty)(0<a<+\infty)$.

Theorem 3.1 Iff is continuous, then the problem (1.1) has at least one solution.

Proof Define a set by

$$
\Omega:=\left\{u, u^{\prime} \in X:\|u\|<A+a T,\left\|u^{\prime}\right\|<a\right\} .
$$

From the definition of $\phi^{-1}:(-\infty,+\infty) \rightarrow(-a, a),(2.2),(2.7)$, and (2.8), we get, for any $u \in \Omega$,

$$
\begin{aligned}
\|S u\| & =\max _{t \in[0, T]}|(S u)(t)| \\
& =\max _{t \in[0, T]}\left|A+\int_{0}^{t} \phi^{-1}((B x)(s)) d s\right| \leq A+a T
\end{aligned}
$$

and

$$
\left\|(S u)^{\prime}\right\|=\max _{t \in[0, T]}\left|(S u)^{\prime}(t)\right|=\max _{t \in[0, T]}\left|\phi^{-1}((B x)(t))\right| \leq a .
$$

Thus $S: \bar{\Omega} \rightarrow \bar{\Omega}$. Utilizing (2.9) and Arzela-Ascoli theorem, it is easy to verify that the nonlinear operator $S$ is a completely continuous operator. Therefore, the nonlinear operator $S$ has at least one fixed point by Schauder fixed point theorem.

From the definition of $S$ in (2.8), we have

$$
\begin{aligned}
& (S u)(0)=A, \\
& (S u)^{\prime}(t)=\phi^{-1}((B u)(t)),
\end{aligned}
$$

i.e.,

$$
\phi\left((S u)^{\prime}(t)\right)=(B u)(t) .
$$

Then we obtain

$$
\begin{aligned}
\phi\left((S u)^{\prime}(T)\right) & =(B u)(T) \\
& =A \sum_{i=1}^{k+1} \tau_{i}+\sum_{i=1}^{k+1} \tau_{i} \int_{0}^{\zeta_{i}} u^{\prime}(s) d s \\
& =\tau u(T)+\sum_{i=1}^{k} \tau_{i} u\left(\zeta_{i}\right)+\sum_{i=1}^{k+1} \tau_{i}[u(0)-A]
\end{aligned}
$$


and

$$
\left(\phi\left((S u)^{\prime}(t)\right)\right)^{\prime}=-f\left(t, u(t), u^{\prime}(t)\right) .
$$

Consequently, we conclude that the fixed point of $S$ is a solution of the problem (1.1).

Remark 3.2 Theorem 3.1 shows that if $\phi$ is singular $(a<\infty)$ and $f$ is continuous on $[0, T] \times \mathbb{R}^{2}$, then the problem (1.1) is always solvable.

Case II. Bounded $\phi$-Laplacian operator: $\phi:(-a, a) \rightarrow(-b, b), 0<b<+\infty$.

Theorem 3.2 If the conditions (i)-(iv) hold, then the nonlinear operator B defined by (2.7) has at least one fixed point. Further, the problem (1.1) has at least one positive solution.

Proof Define a set by

$$
\Omega_{b}:=\{x \in X:\|x\|<b\} .
$$

From the conditions (ii) and (iv), we obtain, for any $x \in P \cap \Omega_{b}$,

$$
\begin{aligned}
(B x)(t)= & \Delta+\sum_{i=1}^{k+1} \tau_{i} \int_{0}^{\zeta_{i}} \phi^{-1}(x(s)) d s \\
& +\int_{t}^{T} f\left(s, A+\int_{0}^{s} \phi^{-1}(x(\tau)) d \tau, \phi^{-1}(x(s))\right) d s \\
\geq & 0 .
\end{aligned}
$$

Clearly, the nonlinear operator $B: P \cap \Omega_{b} \rightarrow P$ is well defined.

The condition (iii) implies that we find a constant $r_{2}$ such that

$$
\Delta+\lambda \phi^{-1}\left(r_{2}\right)+M T \leq r_{2}<b .
$$

Define a set by

$$
\Omega_{2}:=\left\{x \in X:\|x\|<r_{2}\right\}
$$

In virtue of the increasing property of $\phi^{-1}$, we get, for any $x \in P \cap \partial \Omega_{2}$,

$$
\begin{aligned}
\|B x\|= & (B x)(0) \\
= & \Delta+\sum_{i=1}^{k+1} \tau_{i} \int_{0}^{\zeta_{i}} \phi^{-1}(x(s)) d s \\
& +\int_{0}^{T} f\left(s, A+\int_{0}^{s} \phi^{-1}(x(\tau)) d \tau, \phi^{-1}(x(s))\right) d s \\
\leq & \Delta+\lambda \phi^{-1}\left(r_{2}\right)+M T \\
\leq & r_{2}=\|x\| .
\end{aligned}
$$


Thus, for any $x \in P \cap \partial \Omega_{2}$, we have

$$
\|B x\| \leq\|x\|
$$

We choose a small positive constant $r_{1}$ such that $r_{1} \leq \min \left\{\Delta, \frac{r_{2}}{2}\right\}$ and define $\Omega_{1}:=\{x \in$ $\left.X:\|x\|<r_{1}\right\}$. Then for any $x \in P \cap \partial \Omega_{1}$, we find

$$
\begin{aligned}
\|B x\|= & (B x)(0) \\
= & \Delta+\sum_{i=1}^{k+1} \tau_{i} \int_{0}^{\zeta_{i}} \phi^{-1}(x(s)) d s \\
& +\int_{0}^{T} f\left(s, A+\int_{0}^{s} \phi^{-1}(x(\tau)) d \tau, \phi^{-1}(x(s))\right) d s \\
\geq & \Delta+\sum_{i=1}^{k+1} \tau_{i} \int_{0}^{\zeta_{i}} \phi^{-1}(x(s)) d s \\
\geq & r_{1}=\|x\| .
\end{aligned}
$$

Thus, for any $x \in P \cap \partial \Omega_{1}$, we get

$$
\|B x\| \geq\|x\| .
$$

In addition, a standard argument involving the Arzela-Ascoli theorem implies that $B$ : $P \cap\left(\bar{\Omega}_{2} \backslash \Omega_{1}\right) \rightarrow P$ is a completely continuous operator. Therefore, the nonlinear operator $B$ has at least one fixed point by the use of Lemma 2.1. Let $x \in P \cap\left(\bar{\Omega}_{2} \backslash \Omega_{1}\right)$ be a fixed point of $B$, then, from (2.6), we obtain

$$
u(t)=A+\int_{0}^{t} \phi^{-1}(x(s)) d s
$$

Consequently, we get from Remark 2.1 that the problem (1.1) has a positive solution $u(t)$.

Remark 3.3 In order to prove the existence of a positive solution of the problem (1.1), we make a change of variable and introduce a first-order differential equation, and investigate the existence of a fixed point of the corresponding nonlinear operator $B$. The technique can be used for the different domains and ranges of $\phi^{-1}$ and give an a priori estimate of the solution.

Theorem 3.3 If $\tau_{1}=\tau_{2}=\cdots=\tau_{k}=\tau=0$ and $f$ satisfies the condition

$$
|f(t, u, v)|<\frac{b}{T}
$$

for any $(t, u, v) \in[0, T] \times[A-a T, A+a T] \times(-a, a)$, then the problem (1.1) has at least one solution.

Proof Adopting a similar technique to (2.2)-(2.6), we define a nonlinear operator $\mathfrak{B}$ by

$$
(\mathfrak{B} x)(t):=\int_{t}^{T} f\left(s, A+\int_{0}^{s} \phi^{-1}(x(\tau)) d \tau, \phi^{-1}(x(s))\right) d s .
$$


Then we get, for any $x \in \Omega_{b}$,

$$
\begin{aligned}
\|\mathfrak{B} x\| & =\max _{t \in[0, T]}|(\mathfrak{B} x)(t)| \\
& \leq \int_{0}^{T}\left|f\left(s, A+\int_{0}^{s} \phi^{-1}(x(\tau)) d \tau, \phi^{-1}(x(s))\right)\right| d s \\
& <b .
\end{aligned}
$$

Then the operator $\mathfrak{B}: \Omega_{b} \rightarrow \Omega_{b}$ has at least one fixed point by the use of Schauder fixed point theorem. Applying expression (2.6), we conclude that the problem (1.1) has at least one solution.

Remark 3.4 Observe that the solution provided by Theorem 3.3 could be trivial or negative.

Theorem 3.4 If the conditions (ii) and (iv) hold and $f$ satisfies the condition

$$
f(t, u, v) \geq \frac{b}{T}
$$

for any $(t, u, v) \in[0, T] \times[A-a T, A+a T] \times(-a, a)$, then problem (1.1) has no solution.

Proof Taking arbitrarily $x \in \Omega_{b}$, we get from (3.2)

$$
\begin{aligned}
(B x)(0)= & \Delta+\sum_{i=1}^{k+1} \tau_{i} \int_{0}^{\zeta_{i}} \phi^{-1}(x(s)) d s \\
& +\int_{0}^{T} f\left(s, A+\int_{0}^{s} \phi^{-1}(x(\tau)) d \tau, \phi^{-1}(x(s))\right) d s \\
> & \int_{0}^{T} f\left(s, A+\int_{0}^{s} \phi^{-1}(x(\tau)) d \tau, \phi^{-1}(x(s))\right) d s \\
\geq & b .
\end{aligned}
$$

Thus, it implies that there exists a neighborhood $N_{\delta}=[0, \delta)$ such that $(B x)(t)>b$ for any $t \in[0, \delta)$. This implies that the nonlinear operator $(S u)(t)=A+\int_{0}^{t} \phi^{-1}((B x)(s)) d s$ is not well defined, since the domain of $\phi^{-1}$ is the interval $(-b, b)$ and thus a solution of (1.1) cannot exist.

Example 3.1 We consider the following nonhomogeneous boundary value problem with $\phi$-Laplacian operator:

$$
\left\{\begin{array}{l}
\left(\phi\left(u^{\prime}(t)\right)\right)^{\prime}=-f\left(t, u(t), u^{\prime}(t)\right), \quad t \in(0,1), \\
u(0)=1, \quad \phi\left(u^{\prime}(1)\right)=\frac{1}{8} u(1)+\frac{1}{2} u\left(\frac{3}{20}\right)-\frac{1}{8} u\left(\frac{1}{5}\right),
\end{array}\right.
$$

where

$$
\begin{aligned}
& \phi(x)=\frac{5 x}{\sqrt{25+x^{2}}}, \\
& f(t, u, v)=\frac{t}{2}|\sin u|+t|\cos v| .
\end{aligned}
$$


It is easy to see that the nonlinearity $f$ satisfies the condition (i), that is,

$$
0 \leq f(t, u, v) \leq \frac{3}{2}
$$

for any $(t, u, v) \in[0,1] \times(-\infty,+\infty) \times(-\infty,+\infty)$. Computation yields

$$
\Delta=A \sum_{i=1}^{k+1} \tau_{i}=\frac{1}{2}
$$

and there exists a positive constant $3 \leq r \leq 4.683$ such that the following inequalities hold:

$$
\frac{1}{2}+\frac{1}{5} \phi^{-1}(r)+\frac{3}{2} \leq r<5
$$

and

$$
\min _{0 \leq v(t)<5} \sum_{i=1}^{3} \tau_{i} \int_{0}^{\zeta_{i}} \phi^{-1}(v(s)) d s \geq 0
$$

Then conditions (ii)-(iv) also hold. Therefore, we find from Theorem 3.2 that the differential equation (3.3) has at least one positive solution.

\section{Competing interests}

The authors declare that they have no competing interests.

\section{Authors' contributions}

All authors contributed significantly in writing this paper. All authors read and approved the final manuscript.

\section{Author details}

${ }^{1}$ Department of Mathematics, Ningbo University, Ningbo, 315211, P.R. China. ${ }^{2}$ Basic Science Department, Tianjin Agricultural University, Tianjin, 300384, P.R. China.

\section{Acknowledgements}

The authors would wish to express their appreciations to the anonymous referee for his/her valuable suggestions, which have greatly improved this paper. The work was partially supported by NSFC of China (No. 11201248), K.C. Wong Fund of Ningbo University.

Received: 13 October 2013 Accepted: 1 April 2014 Published: 10 Apr 2014

\section{References}

1. Bereanu, C, Mawhin, J: Existence and multiplicity results for some nonlinear equations with singular $\phi$-Laplacian. J. Differ. Equ. 243, 536-557 (2007)

2. Bereanu, C, Mawhin, J: Boundary value problems for some nonlinear systems with singular $\phi$-Laplacian. J. Fixed Point Theory Appl. 4, 57-75 (2008)

3. Bereanu, C, Mawhin, J: Nonhomogeneous boundary value problems for some nonlinear equations with singular $\phi$-Laplacian. J. Math. Anal. Appl. 352, 218-233 (2009)

4. Torres, PJ: Nondegeneracy of the periodically forced Liénard differential equation with $\phi$-Laplacian. Commun. Contemp. Math. 13, 283-292 (2011)

5. Wang, Y, Hou, C: Existence of multiple positive solutions for one-dimensional p-Laplacian. J. Math. Anal. Appl. 315 , 114-153 (2006)

6. Webb, JRL, Lan, KQ: Eigenvalue criteria for existence of multiple positive solutions of nonlinear boundary value problems of local and nonlocal type. Topol. Methods Nonlinear Anal. 27, 91-115 (2006)

7. Webb, JRL, Infante, G: Positive solutions of nonlocal boundary value problems: a unified approach. J. Lond. Math. Soc. 74, 673-693 (2006)

8. Krasnosel'skii, MA: Positive Solutions of Operator Equations. Noordhoff, Groningen (1964)

10.1186/1687-2770-2014-82

Cite this article as: Chen and Ma: The solvability of nonhomogeneous boundary value problems with $\phi$-Laplacian operator. Boundary Value Problems 2014, 2014:82 